\title{
含取代噁唑环的新型吡唑酰胺类化合物的合成与生物活性研究
}

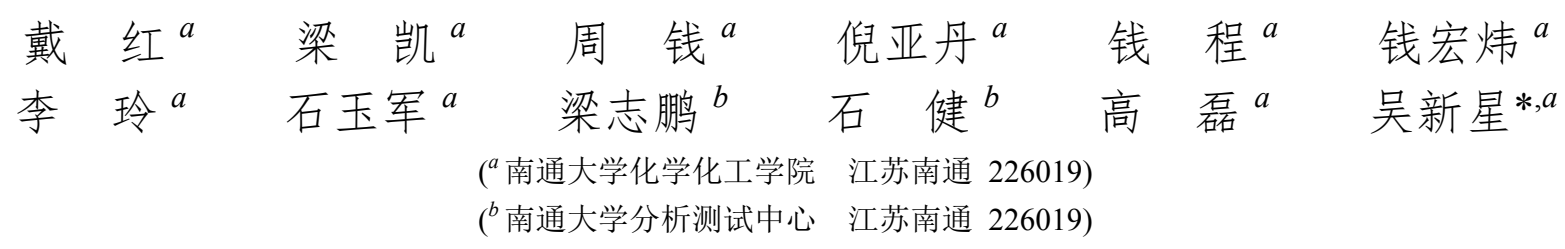

\begin{abstract}
摘要 采用活性单元拼接的方法, 以新型吡唑酰胺类杀螨剂吡螨胺为先导结构, 在吡唑酰胺分子结构中引入取代噁唑 活性单元, 设计合成出 15 个结构新颖的含取代噁夾环的吡唑酰胺类衍生物. 目标化合物的结构通过 ${ }^{1} \mathrm{H} N \mathrm{NR},{ }^{13} \mathrm{C} N \mathrm{NR}$ 和元素分析等进行了表征. 初步室内杀虫活性测试结果表明, 在 $500 \mu \mathrm{g} / \mathrm{mL}$ 测试浓度下, 多数目标化合物对粘虫 (Oriental armyworm) 有着较高的杀虫活性(致死率 $\geqslant 90 \%$ ). 其中 1 个目标化合物在 $500 \mu \mathrm{g} / \mathrm{mL}$ 测试浓度下对蚜虫(Aphis medicaginis)的致死率达 $100 \%$. 此外, 2 个目标化合物在 $500 \mu \mathrm{g} / \mathrm{mL}$ 测试浓度下对朱砂叶螨(Tetranychus cinnabarinus)的 致死率都为 $30 \%$.
\end{abstract}

关键词 噁唑; 吡唑酰胺; 合成; 生物活性

\section{Synthesis and Biological Activities of Novel Pyrazole Amide Compounds Containing Substituted Oxazole Unit}

\author{
Dai, Hong ${ }^{a}$ \\ Liang, $\mathrm{Kai}^{a}$ \\ Zhou, Qian ${ }^{a}$ \\ Ni, $\operatorname{Yadan}^{a}$ \\ Qian, Cheng ${ }^{a}$ \\ Qian, Hongwei ${ }^{a}$ \\ Li, Ling ${ }^{a}$ \\ Shi, Yujun ${ }^{a}$ \\ Liang, Zhipeng ${ }^{b} \quad$ Shi, Jian ${ }^{b}$ \\ Gao, Lei ${ }^{a}$ \\ $\mathrm{Wu}$, Xinxing ${ }^{*, a}$ \\ ( ${ }^{a}$ College of Chemistry and Chemical Engineering, Nantong University, Nantong, Jiangsu 226019) \\ ( ${ }^{b}$ Analysis and Testing Center, Nantong University, Nantong, Jiangsu 226019)
}

\begin{abstract}
In search of new pyrazole amide derivatives with wonderful bioactivities, fifteen novel pyrazole amide compounds were designed and synthesized by introducing substituted oxazole unit into pyrazole amide skeleton based on the lead compound tebufenpyrad. The structures of the title compounds were confirmed by ${ }^{1} \mathrm{H} N M R,{ }^{13} \mathrm{C}$ NMR and elemental analysis. The preliminary bioassay data exhibited that most of target compounds had more than $90 \%$ insecticidal activities against Oriental armyworm at the concentration of $500 \mu \mathrm{g} / \mathrm{mL}$. At the concentration of $500 \mu \mathrm{g} / \mathrm{mL}$, one compound displayed $100 \%$ mortality rate against Aphis medicaginis. In addition, two compounds showed 30\% insecticidal activity against Tetranychus cinnabarinus at the concentration of $500 \mu \mathrm{g} / \mathrm{mL}$.

Keywords oxazole; pyrazole amide; synthesis; biological activity
\end{abstract}

近年来，含氮杂环由于其具有广谱的生物活性而引 起众多科研工作者的关注. 噁唑类化合物作为含氮杂环 家族中重要一员, 在生物学领域展现出优良的杀虫、杀 菌、除草、抗病毒和抗肿瘤等活性 ${ }^{[1-8]}$. 如徐风波课题 组 ${ }^{[9]}$ 报道的化合物 $\mathbf{A}$ (图 1)在 $50 \mathrm{mg} / \mathrm{L}$ 浓度下对粘虫表 现出 $100 \%$ 的杀虫效果; 石玉军等 ${ }^{[10]}$ 研发的化合物 B (图 1)在测试剂量为 $1500 \mathrm{~g} / \mathrm{ha}$ 下对芥菜、小㵞和酸模都有
$100 \%$ 的除草效果; 周钱等 ${ }^{[11]}$ 合成的化合物 $\mathbf{C}$ (图 1)在 $500 \mu \mathrm{g} / \mathrm{mL}$ 浓度下对朱砂叶螨、蚜虫和粘虫的杀虫活性 分别为 $100 \%, 100 \%$ 和 $80 \%$. 吡唑酰胺亦为一类重要的 含氮杂环衍生物，因其良好的杀虫、杀菌、抗癌等活性 在农药、医药等领域具有广泛的应用 ${ }^{[12-20]}$. 如日本三菱 化学公司先后开发的吡螨胺(Tebufenpyrad, 图 1)和唑虫 酰胺(Tolfenpyrad，图 1)具有杀虫谱广、药效快等特点，

\footnotetext{
* Corresponding author. E-mail: wuxinxng@163.com.

Received July 9, 2020; revised July 28, 2020; published online July 30, 2020.

Project supported by the National Natural Science Foundation of China (No. 21372135), the Science and Technology Project Fund of Nantong City (No. MS12019060) and the Program of High-Level Talents of Nantong University (No. 03083031).

国家自然科学基金(No. 21372135)、南通市科技计划(No. MS12019060)和南通大学高层次人才(No. 03083031)资助项目.
} 
对螨虫、小菜蛾等害虫展现优异的防治作用 ${ }^{[21-22]}$; 美国 DuPont 公司研制的氯虫酰胺(Chlorantraniliprole, 图 1) 和氰虫酰胺(Cyantraniliprole, 图 1)对粘虫、甜菜夜蛾和 烟粉同等害虫均有优良的杀虫活性 ${ }^{[23]} ; \mathrm{Li}$ 课题组 ${ }^{[24]}$ 研究 发现化合物 $\mathbf{D}$ (图 1)呈现出良好的杀虫效果, 在 $10 \mathrm{mg} / \mathrm{L}$ 浓度下对小菜蛾和粘虫的致死率均达 $100 \%$, Wang 等 ${ }^{[25]}$ 报道化合物 $\mathbf{E}$ (图 1)显示出较好的杀菌与杀虫效果, 在 $50 \mu \mathrm{g} / \mathrm{mL}$ 浓度下对苹果轮纹病菌的抑菌率为 $89.7 \%$, 在 $200 \mu \mathrm{g} / \mathrm{mL}$ 浓度下对粘虫有 $100 \%$ 的致死率. 因此, 为了 继续从吡唑酰胺衍生物中探索出具有优良生物活性的 物质, 本工作以杀螨剂吡螨胺为农药先导, 通过取代噁 唑活性单元代替吡螨胺结构中的取代苯环片段, 设计并 制备出了一系列含取代噁唑结构的吡唑酰胺化合物(图 2), 同时测定了所合成化合物对粘虫、蚜虫和朱砂叶螨 的生物活性. 目标化合物合成路线见 Scheme 1.

\section{1 结果与讨论}

\section{1 目标化合物的合成}

在制备目标化合物 11 时, 以化合物 11a 为例, 尝试 了不同的缚酸剂和溶剂对 11a 单步收率的影响(表 1). 在 研究过程中发现, 最初以吡啶为缚酸剂, 以二氯甲烷为 溶剂, 室温反应 $10 \mathrm{~h}$, 化合物 $11 \mathrm{a}$ 收率为 $33 \%$, 薄层色谱 (TLC) 跟踪显示有部分原料存在, 然后选用四氢呋喃、乙 腈或甲苯作溶剂, 室温反应 $10 \mathrm{~h}$, 化合物 $10 \mathrm{a}$ 收率分别
为 $35 \%, 38 \%$ 和 $0 \%$. 同样以吡啶为缚酸剂, 以四氢呋喃、 乙腈为溶剂, 将室温反应改成加热回流反应, TLC 检测 表明，大部分原料仍未反应，化合物 11a 的收率分别为 $16 \%$ 和 $24 \%$, 要低于室温反应的收率. 随后改用三乙胺 为缚酸剂, 分别以二氯甲烷、四氢呋喃和乙腈作溶剂, 室温反应 $10 \mathrm{~h}$, 化合物 $11 \mathrm{a}$ 收率分别为 $48 \%, 52 \%$ 和 $21 \%$, 其中以二氯甲烷或四氢呋喃作溶剂的收率比吡啶 作缚酸剂时的收率有了一定的提高. 后来选用碳酸钾为 缚酸剂, 四氢呋喃为溶剂, 室温反应 $10 \mathrm{~h}, \mathrm{TLC}$ 检测发 现仍有部分原料存在，化合物 11a 收率较低，仅为 $32 \%$. 因此, 选以三乙胺为缚酸剂, 四氢呋喃为反应溶剂, 室 温反应 $10 \mathrm{~h}$ 是制备目标化合物的较好方法. 采用该方法 顺利地制备出其它含取代噁唑结构的吡唑酰胺.

\section{2 目标化合物 11 的谱图分析}

以目标化合物 $11 b$ 为例展开分析. 从 $11 b$ 的 ${ }^{1} \mathrm{H}$ NMR 谱图数据而知, 4-F 取代苯环上 2 个氢在 $\delta 7.59 \sim 7.62$ 以 多重峰出现, 酰胺键上氢在 $\delta 7.45$ 以单峰出现, 取代啞 唑环上 4-位氢在 $\delta 7.24$ 以单峰出现, 4-F 取代苯环上另外 2 个氢在 $\delta 7.10 \sim 7.14$ 以多重峰出现, 与酰胺键相连的 亚甲基上 2 个氢因氮原子和噁唑环的诱导效应偏向低 场, 在 $\delta 4.81$ 处以双重峰出现, 取代吡坐环 1-位甲基上 3 个氢因氮原子的诱导作用进而向低场移动, 在 $\delta 4.14$ 处 以单峰出现, 取代吡唑环 3-位甲基 3 个氢在 $\delta 2.25$ 处以 单峰出现. 由 $11 b$ 的 ${ }^{13} \mathrm{C} \mathrm{NMR}$ 谱图数据可知, 酰胺键<smiles>CONC(=O)c1cc(Cl)cc(C)c1NC(=O)c1coc(-c2ccccc2Br)n1</smiles>

A<smiles>CCc1nn(C)c(C(=O)NCc2ccc(C(C)(C)C)cc2)c1Cl</smiles><smiles>CNC(=O)c1cc(C#N)cc(C)c1NC(=O)c1cc(Br)nn1-c1ncccc1Cl</smiles>

Cyantraniliprole
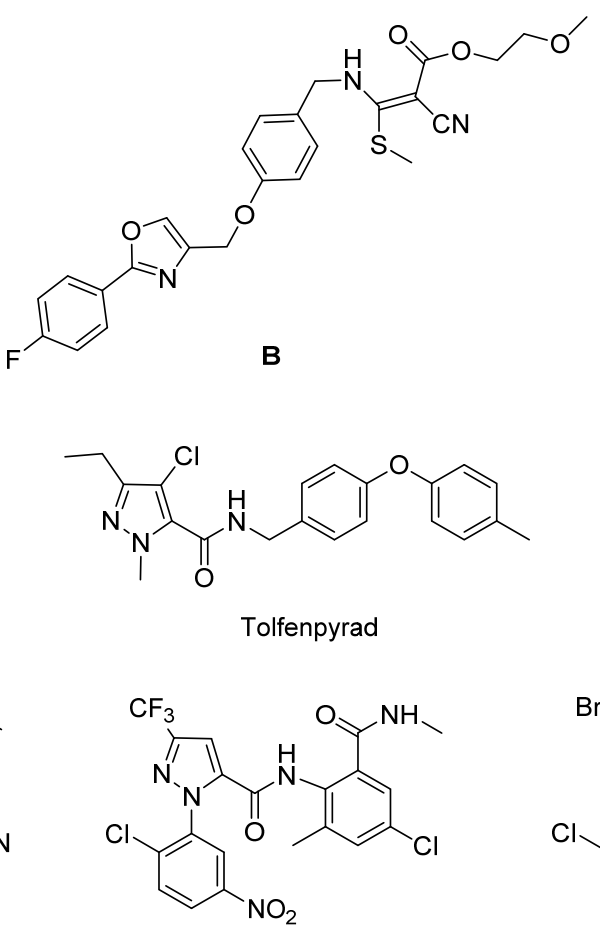

D<smiles>Cc1nn(C)c(Oc2ccc(OC(F)(F)F)cc2)c1/C=N/OCc1ccc(-c2cnco2)cc1</smiles>

C<smiles>CNC(=O)c1cc(Cl)cc(C)c1NC(=O)c1cc(Br)nn1-c1ncccc1Cl</smiles>

Chlorantraniliprole

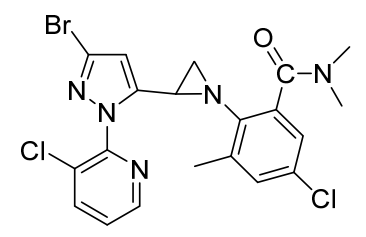

E

图 1 化合物 $\mathbf{A} \sim \mathbf{E}$ 、吡螨胺、唑虫酰胺、氯虫酰胺和氰虫酰胺的结构式

Figure 1 Structures of compounds $\mathbf{A} \sim \mathbf{E}$, tebufenpyrad, tolfenpyrad, chlorantraniliprole and cyantraniliprole 


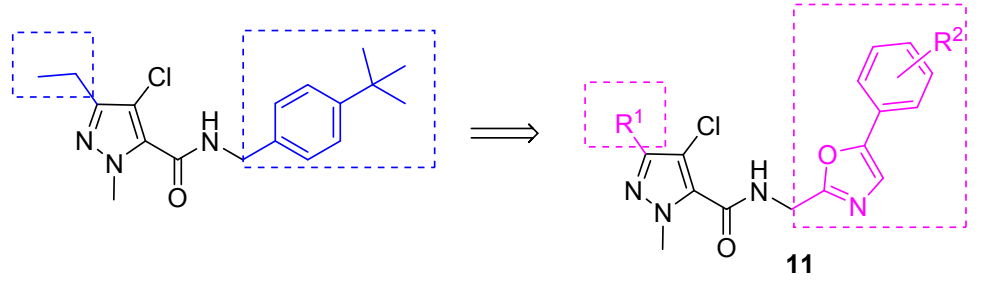

图 2 目标化合物 $\mathbf{1 1}$ 的分子设计图

Figure 2 Design strategy of the title compound 11<smiles>[R1]c1cc(C(=O)OCC)[nH]n1</smiles><smiles>[R]c1nn(C)c(C(=O)OCC)c1Cl</smiles><smiles>[R]c1nn(C)c(C(=O)O)c1Cl</smiles><smiles>[R1]c1nn(C)c(C(=O)Cl)c1Cl</smiles><smiles>NCC(=O)c1ccccc1</smiles><smiles>CCNCCC(Cl)(Cl)Cl</smiles><smiles>[R]C1CCCC1C(=O)CNC(=O)CCl</smiles><smiles>CCc1ncc(-c2cc[R1]cc2)o1</smiles><smiles>CC(C)C1(C)C(=O)N(C)C(=O)c2ccccc21</smiles><smiles>[R]C1CC=C(c2cnc(CN3C(=O)c4ccccc4C3=O)o2)CC1</smiles><smiles></smiles><smiles>[R2]C1C=CC(c2cnc(CN)o2)=CC1</smiles><smiles>[R]c1cc(C(=O)NCc2ncc(-c3[R1]c4c(Cl)c([R2])cc4cc3)o2)n(C)n1</smiles>

11a: $R^{1}=\mathrm{CH}_{3}, \mathrm{R}^{2}=\mathrm{H} ; 11 \mathrm{~b}: \mathrm{R}^{1}=\mathrm{CH}_{3}, \mathrm{R}^{2}=4-\mathrm{F} ; 11 \mathrm{c}: \mathrm{R}^{1}=\mathrm{CH}_{3}, \mathrm{R}^{2}=4-\mathrm{Cl} ; 11 \mathrm{~d}: \mathrm{R}^{1}=\mathrm{CH}_{3}, \mathrm{R}^{2}=4-\mathrm{Br}$;1e: $\mathrm{R}^{1}=\mathrm{C}_{2} \mathrm{H}_{5}, \mathrm{R}^{2}=\mathrm{H} ; 11 \mathrm{f}: \mathrm{R}^{1}=\mathrm{C}_{2} \mathrm{H}_{5}, \mathrm{R}^{2}=$ 4-F; 11g: $\mathrm{R}^{1}=n-\mathrm{C}_{3} \mathrm{H}_{7}, \mathrm{R}^{2}=4-\mathrm{F} ; 11 \mathrm{~h}: \mathrm{R}^{1}=n-\mathrm{C}_{3} \mathrm{H}_{7}, \mathrm{R}^{2}=4-\mathrm{Cl} ; 11 \mathrm{i}: \mathrm{R}^{1}=4-\mathrm{OCH}_{3} \mathrm{C}_{6} \mathrm{H}_{4}, \mathrm{R}^{2}=\mathrm{H} ; 11 \mathrm{j}: \mathrm{R}^{1}=4-\mathrm{OCH}_{3} \mathrm{C}_{6} \mathrm{H}_{4}, \mathrm{R}^{2}=4-\mathrm{F} ; 11 \mathrm{k}: \mathrm{R}^{1}=4-\mathrm{OCH}_{3}$ $\mathrm{C}_{6} \mathrm{H}_{4}, \mathrm{R}^{2}=4-\mathrm{Cl} ; 11 \mathrm{l}: \mathrm{R}^{1}=4-\mathrm{FC}_{6} \mathrm{H}_{4}, \mathrm{R}^{2}=4-\mathrm{F} ; 11 \mathrm{~m}: \mathrm{R}^{1}=2-\mathrm{ClC}_{6} \mathrm{H}_{4}, \mathrm{R}^{2}=4-\mathrm{Cl} ; 11 \mathrm{n}: \mathrm{R}^{1}=2,4-\mathrm{F}_{2} \mathrm{C}_{6} \mathrm{H}_{3}, \mathrm{R}^{2}=\mathrm{H} ; 11 \mathrm{o}: \mathrm{R}^{1}=2,4-\mathrm{Cl}_{2} \mathrm{C}_{6} \mathrm{H}_{3}, \mathrm{R}^{2}=4-\mathrm{F}$

图式 1 目标化合物 11 的合成路线

Scheme 1 Synthetic route of the target compound 11

表 1 反应条件对化合物 11a 收率的影响

Table 1 Effects of reaction conditions on the synthesis of compound 11a

\begin{tabular}{|c|c|c|c|c|}
\hline Entry & Base & Solvent & Reaction condition & Yield/\% \\
\hline 1 & Pyridine & $\mathrm{CH}_{2} \mathrm{Cl}_{2}$ & Room temperature for $10 \mathrm{~h}$ & 33 \\
\hline 2 & Pyridine & THF & Room temperature for $10 \mathrm{~h}$ & 35 \\
\hline 3 & Pyridine & $\mathrm{CH}_{3} \mathrm{CN}$ & Room temperature for $10 \mathrm{~h}$ & 38 \\
\hline 4 & Pyridine & Toluene & Room temperature for $10 \mathrm{~h}$ & 0 \\
\hline 5 & Pyridine & THF & Reflux for $10 \mathrm{~h}$ & 16 \\
\hline 6 & Pyridine & $\mathrm{CH}_{3} \mathrm{CN}$ & Reflux for $10 \mathrm{~h}$ & 24 \\
\hline 7 & $\mathrm{Et}_{3} \mathrm{~N}$ & $\mathrm{CH}_{2} \mathrm{Cl}_{2}$ & Room temperature for $10 \mathrm{~h}$ & 48 \\
\hline 8 & $\mathrm{Et}_{3} \mathrm{~N}$ & THF & Room temperature for $10 \mathrm{~h}$ & 52 \\
\hline 9 & $\mathrm{Et}_{3} \mathrm{~N}$ & $\mathrm{CH}_{3} \mathrm{CN}$ & Room temperature for $10 \mathrm{~h}$ & 21 \\
\hline 10 & $\mathrm{~K}_{2} \mathrm{CO}_{3}$ & THF & Room temperature for $10 \mathrm{~h}$ & 32 \\
\hline
\end{tabular}

$\mathrm{C}=\mathrm{O}$ 碳原子的 $\delta$ 值为 158.5 , 与酰胺键相连的亚甲基碳 原子的 $\delta$ 值为 40.8, 取代吡唑环 1-位甲基碳原子的 $\delta$ 值 为 36.9, 取代吡唑环 3-位甲基碳原子的 $\delta$ 值为 11.1 .

\section{3 生物活性}

利用浸叶法研究目标化合物 $11 \mathbf{a} \sim 110$ 杀粘虫 (Oriental armyworm) 效果, 并通过喷雾法测试目标化合 物 11a 110 对蚜虫(Aphis medicaginis) 及朱砂叶螨
(Tetranychus cinnabarinus)的杀虫活性 ${ }^{[26]}$. 由表 2 可看 出, 在 $500 \mu \mathrm{g} / \mathrm{mL}$ 测试浓度下, 大多数目标化合物对粘 虫显示出良好的杀虫效果，其中化合物 $11 \mathrm{a} \sim 11 \mathrm{11} 、 1 \mathrm{n}$ 和 110 对粘虫的致死率为 $90 \% \sim 100 \%$ ，与对照药剂唑虫 酰胺的杀虫活性相当. 部分目标化合物在测试浓度降至 $100 \mu \mathrm{g} / \mathrm{mL}$ 时对粘虫仍展现出一定的杀虫活性，化合物

11e、11g 和 110 对粘虫的致死率分别为 $30 \%, 30 \%$ 和 $40 \%$, 其中化合物 110 对粘虫的杀虫效果与对照药唑虫酰胺相 接近. 通过构效关系可以看出，当 $\mathrm{R}^{1}$ 为烷基时，化合物 11e $\left(\mathrm{R}^{1}=\mathrm{C}_{2} \mathrm{H}_{5}, \mathrm{R}^{2}=\mathrm{H}\right)$ 和 $11 \mathrm{~g}\left(\mathrm{R}^{1}=n-\mathrm{C}_{3} \mathrm{H}_{7}, \mathrm{R}^{2}=4-\mathrm{F}\right)$ 对粘 虫的杀虫活性要高于其它化合物; 当 $\mathrm{R}^{1}$ 为取代苯基时, 化合物 $110\left(\mathrm{R}^{1}=2,4-\mathrm{Cl}_{2} \mathrm{C}_{6} \mathrm{H}_{3}, \mathrm{R}^{2}=4-\mathrm{F}\right)$ 对粘虫的防治作 用要优于化合物 $11 \mathbf{i} \sim 11 \mathrm{n}$. 同时, 在 $500 \mu \mathrm{g} / \mathrm{mL}$ 测试浓 度下，化合物 $110\left(\mathrm{R}^{1}=2,4-\mathrm{Cl}_{2} \mathrm{C}_{6} \mathrm{H}_{3}, \mathrm{R}^{2}=4-\mathrm{F}\right)$ 对蚜虫表 现出良好的杀虫活性，对蚜虫的致死率为 $100 \%$, 要高 于其它目标化合物。此外, 化合物 $11 \mathbf{k}\left(\mathrm{R}^{1}=\right.$ 4- $\left.\mathrm{CH}_{3} \mathrm{O}-\mathrm{C}_{6} \mathrm{H}_{4}, \mathrm{R}^{2}=4-\mathrm{Cl}\right)$ 和 $11 \mathrm{l}\left(\mathrm{R}^{1}=4-\mathrm{FC}_{6} \mathrm{H}_{4}, \mathrm{R}^{2}=4-\mathrm{F}\right)$ 在 $500 \mu \mathrm{g} / \mathrm{mL}$ 测试浓度下对朱砂叶螨均有 $30 \%$ 的杀虫效果, 要好于其它目标化合物的杀虫效果. 目标化合物 11a 110 对朱砂叶螨的杀虫效果不明显, 推测可能是由于化 
表 2 目标化合物 $11 \mathrm{a} \sim 110$ 的杀虫活性(死亡率/\%) ${ }^{a}$

Table 2 Insecticidal activities (mortality/\%) of title compounds 11a $\sim \mathbf{1 1 0}$

\begin{tabular}{|c|c|c|c|c|c|}
\hline \multirow{2}{*}{ Compd. } & \multicolumn{2}{|c|}{ Oriental armyworm } & \multicolumn{2}{|c|}{ Aphis medicaginis } & \multirow{2}{*}{$\begin{array}{c}\text { Tetranychus cinnabarinus } \\
500 \mu \mathrm{g} / \mathrm{mL}\end{array}$} \\
\hline & $500 \mu \mathrm{g} / \mathrm{mL}$ & $100 \mu \mathrm{g} / \mathrm{mL}$ & $500 \mu \mathrm{g} / \mathrm{mL}$ & $100 \mu \mathrm{g} / \mathrm{mL}$ & \\
\hline 11a & 100 & 0 & 0 & - & 0 \\
\hline $11 \mathrm{~b}$ & 100 & 0 & 0 & - & 0 \\
\hline $11 \mathrm{c}$ & 100 & 0 & 0 & - & 0 \\
\hline 11d & 100 & 0 & 0 & - & 0 \\
\hline $11 \mathrm{e}$ & 100 & 30 & 0 & - & 0 \\
\hline 11f & 100 & 0 & 0 & - & 0 \\
\hline $11 \mathrm{~g}$ & 100 & 30 & 0 & - & 0 \\
\hline $11 \mathrm{~h}$ & 100 & 0 & 0 & - & 0 \\
\hline $11 \mathrm{i}$ & 100 & 0 & 0 & - & 0 \\
\hline $11 \mathbf{j}$ & 90 & 0 & 0 & - & 0 \\
\hline $11 \mathrm{k}$ & 100 & 0 & 0 & - & 30 \\
\hline 111 & 100 & 0 & 0 & - & 30 \\
\hline $11 \mathrm{~m}$ & 0 & - & 0 & - & 0 \\
\hline $11 n$ & 100 & 0 & 0 & - & 0 \\
\hline 110 & 100 & 40 & 100 & 0 & 0 \\
\hline Tolfenpyrad & 100 & 50 & - & - & - \\
\hline Imidacloprid & - & - & 100 & 100 & - \\
\hline Tebufenpyrad & - & - & - & - & 100 \\
\hline
\end{tabular}

a “_, refers to not tested.

合物分子中酰胺键部分所连有的噁唑连取代苯基基团 使得空间位阻变大, 进而不利于目标化合物分子与靶标 有机地结合. 以上试验数据表明, 将取代噁唑活性片段 引入到吡唑酰胺骨架中, 得到的一些化合物对粘虫呈现 出良好的杀虫作用.

\section{2 结论}

本研究利用活性单元拼接方法, 合成出了一系列具 有取代噁唑单元结构的新型吡唑酰胺. 通过核磁共振氢 谱、核磁共振碳谱及元素分析等手段对目标化合物进行 了结构确证. 生测数据表明, 在 $500 \mu \mathrm{g} / \mathrm{mL}$ 测试浓度下, 大部分化合物对粘虫的杀死率在 $90 \% \sim 100 \%$ ，当测试 浓度降至 $100 \mu \mathrm{g} / \mathrm{mL}$ 时, 化合物 11e、11g 和 110 对粘虫 仍有 $30 \%, 30 \%$ 和 $40 \%$ 的杀虫活性; 化合物 110 在 500 $\mu \mathrm{g} / \mathrm{mL}$ 测试浓度下对蚜虫的致死率达 $100 \%$; 此外, 化 合物 $11 \mathrm{k}$ 和 111 在 $500 \mu \mathrm{g} / \mathrm{mL}$ 浓度下对朱砂叶螨呈现出 一定的杀虫效果, 对朱砂叶螨的致死率都为 $30 \%$. 这为 今后进一步从事吡唑酰胺杀虫剂的创制研究提供了重 要的参考信息.

\section{3 实验部分}

\section{1 仪器与试剂}

核磁共振氢谱和核磁共振碳谱通过 BRUKER 400 MHz 核磁共振仪 (TMS 为内标) 测定; 化合物的熔点经 X-4 显微熔点测定仪(温度计未经校正)测定; 化合物的 元素分析由 Yanaco-CHN CORDER MT-3 自动元素分析
仪测试. 实验中所用试剂均为分析纯试剂.

\section{2 化合物 1 4 和 $6 \sim 8$ 的合成}

化合物 1 4 参照文献[27]合成, 化合物 $6 \sim 8$ 参照 文献[28]和[29]合成.

\section{3 化合物 5 的合成}

在一反应瓶中加入 $8 \mathrm{mmol}$ 中间体 4 和 $0.4 \mathrm{~mol}$ 氯化 亚砜，室温摚拌片刻后，向其滴加 3 滴 $N, N$-二甲基甲酰 胺(DMF), 接着将反应液升温至回流, 搅拌 $4 \sim 5 \mathrm{~h}$. 反 应结束后, 将反应液冷却至室温并浓缩至干, 得到化合 物 $\mathbf{5}$, 不经纯化直接用于后面的反应.

\section{4 化合物 9 的合成}

在一反应瓶中加入 $20.4 \mathrm{mmol}$ 中间体 4、40.8 $\mathrm{mmol}$ 邻苯二甲酰亚胺钾盐和 $70 \mathrm{~mL}$ DMF, 接着将反应液室 温搅拌 $3 \mathrm{~h}$. 停止反应, 将反应液倒入盛水 $500 \mathrm{~mL}$ 水的 烧杯中, 静置过夜, 析出固体. 通过抽滤、真空干燥后得 到化合物 $\mathbf{9}$, 化合物 9 可直接投入下一步的反应.

为了验证其化合物 9 的结构, 选取其中 $\mathrm{R}^{2}$ 为 4-F 的 化合物以乙醇为溶剂进行了重结晶, 得到黄色固体 $\mathbf{9}$, 产率 72\%. m.p. 153 $155{ }^{\circ} \mathrm{C} ;{ }^{1} \mathrm{H}$ NMR $\left(\mathrm{CDCl}_{3}, 400\right.$ MHz) $\delta: 7.91 \sim 7.93(\mathrm{~m}, 2 \mathrm{H}), 7.75 \sim 7.78(\mathrm{~m}, 2 \mathrm{H}), 7.55 \sim$ $7.58(\mathrm{~m}, 2 \mathrm{H}), 7.19(\mathrm{~s}, 1 \mathrm{H}), 7.07 \sim 7.11(\mathrm{~m}, 2 \mathrm{H}), 5.06(\mathrm{~s}$, $2 \mathrm{H}) ;{ }^{13} \mathrm{C}$ NMR $\left(\mathrm{CDCl}_{3}, 101 \mathrm{MHz}\right) \delta: 167.3,162.6(\mathrm{~d}, J=$ $247.4 \mathrm{~Hz}), 157.7,151.3,134.4,132.0,126.3,126.2,123.9$, 123.8, 121.8, $116.1(\mathrm{~d}, J=22.0 \mathrm{~Hz}), 34.8$. Anal. calcd for $\mathrm{C}_{18} \mathrm{H}_{11} \mathrm{FN}_{2} \mathrm{O}_{3}$ : C 67.08, H 3.44, N 8.69; found C 67.27, H $3.28, \mathrm{~N} 8.83$. 


\section{5 化合物 10 的合成}

在一反应瓶中加入 $9.9 \mathrm{mmol}$ 化合物 9 及 $40 \mathrm{~mL}$ 无 水乙醇, 室温搅拌下向其中加入 $29.6 \mathrm{mmol} 80 \%$ 水合肼, 接着将反应液加热回流反应 $1 \mathrm{~h}$. 反应结束后, 将反应 液冷却至室温, 抽滤, 用乙醇洗涤固体数次, 所得母液 浓缩至干, 得到化合物 $\mathbf{1 0}$, 不经纯化可直接投入后面的 反应.

\section{6 目标化合物 11 的合成}

在一反应瓶中加入 $4.16 \mathrm{mmol}$ 化合物 $10 、 5 \mathrm{mmol}$ 三乙胺和 $20 \mathrm{~mL}$ 四氢呋喃, 冰浴条件下向其中缓慢滴加 $5 \mathrm{mmol}$ 化合物 5 的四氢呋喃溶液 ( $5 \mathrm{~mL}$ ). 滴加好后, 继 续室温摚拌 $6 \sim 12 \mathrm{~h}$. 停止反应, 抽滤, 母液浓缩至干, 所得粗品通过柱层析 [ $V$ (乙酸乙酯) $: V$ (石油醚 $)=1: 3]$ 纯化得目标产物 11a $\sim 110$.

4-氯-1,3-二甲基- $N$-[(5-苯基噁唑-2-基)甲基] $-1 H$-吡 唑-5-甲酰胺(11a): 白色固体, 产率 52\%. m.p. 131 $132{ }^{\circ} \mathrm{C} ;{ }^{1} \mathrm{H}$ NMR $\left(\mathrm{CDCl}_{3}, 400 \mathrm{MHz}\right) \delta: 7.62 \sim 7.64(\mathrm{~m}$, $2 \mathrm{H}), 7.40 \sim 7.46(\mathrm{~m}, 3 \mathrm{H}), 7.32 \sim 7.36(\mathrm{~m}, 1 \mathrm{H}), 7.30(\mathrm{~s}$, $1 \mathrm{H}), 4.82(\mathrm{~d}, J=5.2 \mathrm{~Hz}, 2 \mathrm{H}), 4.14$ (s, 3H), 2.25 (s, 3H); ${ }^{13} \mathrm{C}$ NMR $\left(100 \mathrm{MHz}, \mathrm{CDCl}_{3}\right) \delta: 159.4,158.5,152.2,144.6$, $130.5,129.9,129.0,128.7,128.4,127.5,124.2,121.8$, 109.0, 40.8, 37.0, 11.1. Anal. calcd for $\mathrm{C}_{16} \mathrm{H}_{15} \mathrm{ClN}_{4} \mathrm{O}_{2}$ : C 58.10, H 4.57, N 16.94; found C 58.26, H 4.43, N 16.77.

4-氯-1,3-二甲基- $\mathrm{N}$-\{[5-(4-氟苯基)慧唑-2-基]甲 基\}-1H-吡唑-5-甲酰胺(11b): 白色固体, 产率 47\%. m.p. $117 \sim 119{ }^{\circ} \mathrm{C} ;{ }^{1} \mathrm{H}$ NMR $\left(\mathrm{CDCl}_{3}, 400 \mathrm{MHz}\right) \delta: 7.59 \sim 7.62$ (m, 2H), $7.45(\mathrm{~s}, 1 \mathrm{H}), 7.24(\mathrm{~s}, 1 \mathrm{H}), 7.10 \sim 7.14(\mathrm{~m}, 2 \mathrm{H})$, $4.81(\mathrm{~d}, J=5.2 \mathrm{~Hz}, 2 \mathrm{H}), 4.14$ (s, 3H), 2.25 (s, 3H); ${ }^{13} \mathrm{C}$ NMR $\left(100 \mathrm{MHz}, \mathrm{CDCl}_{3}\right) \delta: 162.8(\mathrm{~d}, J=247.5 \mathrm{~Hz}), 158.5$, $151.4,144.6,130.4,128.9,126.3,123.9,121.5,116.1$ (d, $J=22.0 \mathrm{~Hz}$ ), 109.0, 40.8, 36.9, 11.1. Anal. calcd for $\mathrm{C}_{16} \mathrm{H}_{14} \mathrm{ClFN}_{4} \mathrm{O}_{2}$ : C 55.10, H 4.05, N 16.06; found C 55.26, $\mathrm{H} 3.92$, N 16.20 .

4-氯-1,3-二甲基- $N$ - $\{[5$-(4-氯苯基)噁唑-2-基]甲 基\}-1H-吡唑-5-甲酰胺(11c)：黄色固体，产率 48\%. m.p. $113 \sim 115{ }^{\circ} \mathrm{C} ;{ }^{1} \mathrm{H}$ NMR $\left(\mathrm{CDCl}_{3}, 400 \mathrm{MHz}\right) \delta: 7.56$ (d, $J=$ $8.4 \mathrm{~Hz}, 2 \mathrm{H}), 7.39 \sim 7.45(\mathrm{~m}, 3 \mathrm{H}), 7.29(\mathrm{~s}, 1 \mathrm{H}), 4.82(\mathrm{~d}, J=$ $5.2 \mathrm{~Hz}, 2 \mathrm{H}), 4.14$ (s, 3H), 2.25 (s, 3H); ${ }^{13} \mathrm{C}$ NMR $(100$ $\left.\mathrm{MHz}, \mathrm{CDCl}_{3}\right) \delta: 158.5,151.2,144.7,134.6,131.4,130.4$, 129.3, 128.8, 126.0, 125.5, 122.2, 109.0, 40.7, 36.9, 11.0 . Anal. calcd for $\mathrm{C}_{16} \mathrm{H}_{14} \mathrm{Cl}_{2} \mathrm{~N}_{4} \mathrm{O}_{2}$ : C 52.62, H 3.86, N 15.34; found $\mathrm{C} 52.50, \mathrm{H} 3.70, \mathrm{~N} 15.49$.

4-氯-1,3-二甲基- $N$ - $\{[5$-(4-溴苯基)啞唑-2-基]甲 基\}-1H-吡唑-5-甲酰胺(11d): 黄色固体, 产率 52\%. m.p. 144 $146{ }^{\circ} \mathrm{C} ;{ }^{1} \mathrm{H}$ NMR $\left(\mathrm{CDCl}_{3}, 400 \mathrm{MHz}\right) \delta: 7.56$ (d, $J=$
$8.4 \mathrm{~Hz}, 2 \mathrm{H}), 7.46 \sim 7.50(\mathrm{~m}, 3 \mathrm{H}), 7.31(\mathrm{~s}, 1 \mathrm{H}), 4.82(\mathrm{~d}, J=$ $5.6 \mathrm{~Hz}, 2 \mathrm{H}), 4.14(\mathrm{~s}, 3 \mathrm{H}), 2.25(\mathrm{~s}, 3 \mathrm{H}) ;{ }^{13} \mathrm{C}$ NMR $(100$ $\left.\mathrm{MHz}, \mathrm{CDCl}_{3}\right) \delta: 159.8,158.5,151.3,144.7,132.2,130.4$, 126.55, 125.7, 122.7, 122.4, 109.0, 40.8, 37.0, 11.1. Anal. calcd for $\mathrm{C}_{16} \mathrm{H}_{14} \mathrm{BrClN}_{4} \mathrm{O}_{2}$ : C 46.91, H 3.44, N 13.68; found $\mathrm{C} 46.77, \mathrm{H} 3.59, \mathrm{~N} 13.56$.

4-氯-1-甲基-3-乙基- $N$-[(5-苯基唸唑-2-基)甲基]- $1 H$ 吡唑-5-甲酰胺(11e): 黄色固体, 产率 50\%. m.p. 190 $192{ }^{\circ} \mathrm{C} ;{ }^{1} \mathrm{H}$ NMR $\left(\mathrm{CDCl}_{3}, 400 \mathrm{MHz}\right) \delta: 7.62$ (d, $J=7.2$ $\mathrm{Hz}, 2 \mathrm{H}), 7.40 \sim 7.47(\mathrm{~m}, 3 \mathrm{H}), 7.35(\mathrm{~d}, J=7.2 \mathrm{~Hz}, 1 \mathrm{H})$, 7.30 (s, 1H), 4.83 (d, J=5.2 Hz, 2H), 4.15 (s, 3H), 2.66 (q, $J=7.6 \mathrm{~Hz}, 2 \mathrm{H}), 1.25(\mathrm{t}, J=7.6 \mathrm{~Hz}, 3 \mathrm{H}) ;{ }^{13} \mathrm{C}$ NMR $(100$ $\left.\mathrm{MHz}, \mathrm{CDCl}_{3}\right) \delta: 159.6,158.6,152.2,149.7,130.5,129.0$, $128.8,127.5,124.3,121.8,108.3,40.8,37.0,19.2,12.8$. Anal. calcd for $\mathrm{C}_{17} \mathrm{H}_{17} \mathrm{ClN}_{4} \mathrm{O}_{2}$ : C 59.22, H 4.97, N 16.25; found $\mathrm{C} 59.09$, H 5.13, N 16.10.

4-氯-1-甲基-3-乙基- $N$ - $\{$ [5-(4-氟苯基)噁唑-2-基]甲 基\}-1H-吡唑-5-甲酰胺(11f): 灰色固体, 产率 51\%. m.p. $100 \sim 102{ }^{\circ} \mathrm{C} ;{ }^{1} \mathrm{H}$ NMR $\left(\mathrm{CDCl}_{3}, 400 \mathrm{MHz}\right) \delta: 7.59 \sim 7.62$ (m, 2H), $7.47(\mathrm{~s}, 1 \mathrm{H}), 7.24(\mathrm{~s}, 1 \mathrm{H}), 7.10 \sim 7.14(\mathrm{~m}, 2 \mathrm{H})$, $4.82(\mathrm{~d}, J=5.2 \mathrm{~Hz}, 2 \mathrm{H}), 4.14$ (s, 3H), $2.66(\mathrm{q}, J=7.6 \mathrm{~Hz}$, $2 \mathrm{H}), 1.25(\mathrm{t}, J=7.6 \mathrm{~Hz}, 3 \mathrm{H}) ;{ }^{13} \mathrm{C}$ NMR $\left(100 \mathrm{MHz}, \mathrm{CDCl}_{3}\right)$ $\delta: 162.8(\mathrm{~d}, J=247.8 \mathrm{~Hz}), 159.5,158.6,151.4,149.7$, $130.5,126.3,123.9,121.5,116.1$ (d, $J=22.1 \mathrm{~Hz}), 108.3$, 40.8, 36.9, 19.2, 12.8. Anal. calcd for $\mathrm{C}_{17} \mathrm{H}_{16} \mathrm{ClFN}_{4} \mathrm{O}_{2}$ : C 56.28, H 4.45, N 15.44; found C 56.45, H 4.31, N 15.57.

4-氯-1-甲基-3-正丙基- $N$ - $\{$ [5-(4-氟苯基)噁唑-2-基] 甲基 \}-1H-吡唑-5-甲酰胺(11g)：黄色固体，产率 50\%. m.p. $116 \sim 118{ }^{\circ} \mathrm{C} ;{ }^{1} \mathrm{H}$ NMR $\left(\mathrm{CDCl}_{3}, 400 \mathrm{MHz}\right) \delta: 7.59 \sim$ $7.62(\mathrm{~m}, 2 \mathrm{H}), 7.45(\mathrm{~s}, 1 \mathrm{H}), 7.24(\mathrm{~s}, 1 \mathrm{H}), 7.10 \sim 7.14(\mathrm{~m}$, $2 \mathrm{H}), 4.82(\mathrm{~d}, J=5.2 \mathrm{~Hz}, 2 \mathrm{H}), 4.15(\mathrm{~s}, 3 \mathrm{H}), 2.60$ (t, $J=7.6$ $\mathrm{Hz}, 2 \mathrm{H}), 1.66 \sim 1.71(\mathrm{~m}, 2 \mathrm{H}), 0.97(\mathrm{t}, J=7.6 \mathrm{~Hz}, 3 \mathrm{H}) ;{ }^{13} \mathrm{C}$ NMR (100 MHz, $\left.\mathrm{CDCl}_{3}\right) \delta: 162.8(\mathrm{~d}, J=247.7 \mathrm{~Hz}), 159.5$, $158.6,151.3,148.5,130.4,126.2,126.1,123.9,121.5$, $116.1(\mathrm{~d}, J=22.0 \mathrm{~Hz}), 108.6,40.8,36.9,26.7,21.9,13.8$. Anal. calcd for $\mathrm{C}_{18} \mathrm{H}_{18} \mathrm{ClFN}_{4} \mathrm{O}_{2}$ : C 57.37, H 4.81, N 14.87; found $\mathrm{C} 57.50, \mathrm{H} 4.96, \mathrm{~N} 14.75$.

4-氯-1-甲基-3-正丙基- $N$ - $\{$ [5-(4-氯苯基)噁唑-2-基] 甲基\}-1H-吡唑-5-甲酰胺(11h): 黄色固体，产率 53\%. m.p. $117 \sim 119{ }^{\circ} \mathrm{C} ;{ }^{1} \mathrm{H}$ NMR $\left(\mathrm{CDCl}_{3}, 400 \mathrm{MHz}\right) \delta: 7.54(\mathrm{~d}$, $J=8.8 \mathrm{~Hz}, 2 \mathrm{H}), 7.45(\mathrm{~s}, 1 \mathrm{H}), 7.39$ (d, $J=8.8 \mathrm{~Hz}, 2 \mathrm{H}), 7.29$ (s, 1H), $4.82(\mathrm{~d}, J=5.6 \mathrm{~Hz}, 2 \mathrm{H}), 4.15(\mathrm{~s}, 3 \mathrm{H}), 2.60(\mathrm{t}, J=$ $7.6 \mathrm{~Hz}, 2 \mathrm{H}), 1.64 \sim 1.71(\mathrm{~m}, 2 \mathrm{H}), 0.97$ (t, $J=7.6 \mathrm{~Hz}, 3 \mathrm{H})$; ${ }^{13} \mathrm{C}$ NMR (100 MHz, $\left.\mathrm{CDCl}_{3}\right) \delta: 159.9,158.6,151.2,148.5$, $134.6,131.5,130.4,129.3,128.8,126.0,125.2,122.2$, 
108.7, 40.8, 36.9. 27.7, 21.9, 13.8. Anal. calcd for $\mathrm{C}_{18} \mathrm{H}_{18}$ $\mathrm{Cl}_{2} \mathrm{~N}_{4} \mathrm{O}_{2}$ : C 54.97, $\mathrm{H} 4.61, \mathrm{~N} 14.25$; found C 54.83, H 4.45, $\mathrm{N} 14.38$.

4-氯-1-甲基-3-(4-甲氧基苯基)- $N$ - [ (5-苯基啞唑-2基)甲基]- $1 H$-吡唑-5-甲酰胺(11i)：黄色固体，产率 54\%. m.p. $145 \sim 147{ }^{\circ} \mathrm{C} ;{ }^{1} \mathrm{H}$ NMR $\left(\mathrm{CDCl}_{3}, 400 \mathrm{MHz}\right): \delta 7.28 \sim$ $7.80(\mathrm{~m}, 9 \mathrm{H}), 7.25$ (s, 1H), 6.92 (d, $J=8.4 \mathrm{~Hz}, 1 \mathrm{H}), 4.80$ $(\mathrm{d}, J=5.2 \mathrm{~Hz}, 2 \mathrm{H}), 4.16(\mathrm{~s}, 3 \mathrm{H}), 3.88(\mathrm{~s}, 3 \mathrm{H}) ;{ }^{13} \mathrm{C} \mathrm{NMR}$ $\left(100 \mathrm{MHz}, \mathrm{CDCl}_{3}\right): \delta 159.4,158.4,155.2,152.3,144.9$, $131.9,129.3,129.0,128.8,127.5,127.0,124.3,122.6$, 121.7, 111.9, 107.3, 56.2, 41.3, 37.1. Anal. calcd for $\mathrm{C}_{22} \mathrm{H}_{19} \mathrm{ClN}_{4} \mathrm{O}_{3}$ : C 62.49, $\mathrm{H} 4.53, \mathrm{~N}$ 13.25; found $\mathrm{C} 62.65$, $\mathrm{H} 4.40, \mathrm{~N} 13.39$.

4-氯-1-甲基-3-(4-甲氧基苯基)- $N$ - $\{[5$-(4-氟苯基)啞 唑-2-基]甲基 $\}-1 H$-吡唑-5-甲酰胺(11j)：白色固体，产率 $50 \%$. m.p. $148 \sim 150{ }^{\circ} \mathrm{C} ;{ }^{1} \mathrm{H} \mathrm{NMR}\left(\mathrm{CDCl}_{3}, 400 \mathrm{MHz}\right) \delta$ : $7.53 \sim 7.86(\mathrm{~m}, 6 \mathrm{H}), 7.26(\mathrm{~s}, 1 \mathrm{H}), 7.12(\mathrm{t}, J=8.8 \mathrm{~Hz}, 2 \mathrm{H})$, 6.99 (t, $J=8.8 \mathrm{~Hz}, 1 \mathrm{H}), 4.86$ (d, $J=5.6 \mathrm{~Hz}, 2 \mathrm{H}), 4.23$ (s, $3 \mathrm{H}), 3.95(\mathrm{~s}, 3 \mathrm{H}) ;{ }^{13} \mathrm{C}$ NMR $\left(100 \mathrm{MHz}, \mathrm{CDCl}_{3}\right) \delta: 162.8$ $(\mathrm{d}, J=248.2 \mathrm{~Hz}), 159.4,158.4,155.2,151.5,144.9,131.8$, $130.9,129.3,127.0,126.2,124.2,123.8,122.6,121.4$, 116.1 (d, $J=22.0 \mathrm{~Hz}), 111.9,107.2,56.2$, 41.2, 37.0. Anal. calcd for $\mathrm{C}_{22} \mathrm{H}_{18} \mathrm{ClFN}_{4} \mathrm{O}_{3}$ : C 59.94, H 4.12, N 12.71; found C 59.81, H 4.28, N 12.62.

4-氯-1-甲基-3-(4-甲氧基苯基)- $N$ - $\{[5$-(4-氯苯基)噁 唑-2-基]甲基 $\}-1 H$-吡唑-5-甲酰胺(11k): 白色固体, 产率 47\%. m.p. $143 \sim 145{ }^{\circ} \mathrm{C} ;{ }^{1} \mathrm{H}$ NMR $\left(\mathrm{CDCl}_{3}, 400 \mathrm{MHz}\right) \delta$ : $7.53 \sim 7.86(\mathrm{~m}, 5 \mathrm{H}), 7.40(\mathrm{~d}, J=8.8 \mathrm{~Hz}, 2 \mathrm{H}), 7.31(\mathrm{~s}, 1 \mathrm{H})$, 6.99 (d, $J=8.4 \mathrm{~Hz}, 2 \mathrm{H}), 4.86$ (d, $J=5.6 \mathrm{~Hz}, 2 \mathrm{H}), 4.22$ (s, 3H), 3.95 (s, 3H); ${ }^{13} \mathrm{C}$ NMR (100 MHz, $\left.\mathrm{CDCl}_{3}\right) \delta: 159.8$, $158.5,155.2,151.3,144.9,134.6,132.4,131.4,130.9$, $129.3,128.8,127.0,125.9,125.5,124.2$, 122.6, 122.1, 111.9, 107.3, 56.2, 41.2, 37.0. Anal. calcd for $\mathrm{C}_{22} \mathrm{H}_{18} \mathrm{Cl}_{2} \mathrm{~N}_{4}-$ $\mathrm{O}_{3}$ : C 57.78, H 3.97, N 12.25; found C 57.94, H 3.85, N 12.40 .

4-氯-1-甲基-3-(4-氟苯基)- $N$ - $\{[$ - - (4-氟苯基)啞唑-2基]甲基\}- $1 H$-吡唑-5-甲酰胺(111)：黄色固体，产率 52\%. m.p. $185 \sim 187{ }^{\circ} \mathrm{C} ;{ }^{1} \mathrm{H}$ NMR $\left(\mathrm{CDCl}_{3}, 400 \mathrm{MHz}\right) \delta: 7.79 \sim$ $7.82(\mathrm{~m}, 2 \mathrm{H}), 7.60 \sim 7.63(\mathrm{~m}, 2 \mathrm{H}), 7.56(\mathrm{~s}, 1 \mathrm{H}), 7.11 \sim$ $7.16(\mathrm{~m}, 5 \mathrm{H}), 4.86(\mathrm{~d}, J=5.2 \mathrm{~Hz}, 2 \mathrm{H}), 4.23(\mathrm{~s}, 3 \mathrm{H}) ;{ }^{13} \mathrm{C}$ NMR (100 MHz, $\left.\mathrm{CDCl}_{3}\right) \delta: 163.1(\mathrm{~d}, J=246.8 \mathrm{~Hz}), 162.8$ $(\mathrm{d}, J=247.8 \mathrm{~Hz}), 159.4,158.4,151.5,145.6,131.8,130.9$, $129.5,129.4,128.9,126.9,126.3,126.2,123.8,121.5$, $116.2(\mathrm{~d}, J=22.0 \mathrm{~Hz}), 115.6(\mathrm{~d}, J=21.6 \mathrm{~Hz}), 107.4,41.3$, 37.0. Anal. calcd for $\mathrm{C}_{21} \mathrm{H}_{15} \mathrm{ClF}_{2} \mathrm{~N}_{4} \mathrm{O}_{2}$ : C 58.82, $\mathrm{H} \mathrm{3.53,} \mathrm{N}$
13.07; found C 58.97, H 3.37, N 13.18.

4-氯-1-甲基-3-(2-氯苯基)- $N$ - $\{[5$-(4-氯苯基)啞唑-2基]甲基 $\}-1 H$-吡唑-5-甲酰胺(11m)：黄色固体，产率 54\%. m.p. 153 $155{ }^{\circ} \mathrm{C} ;{ }^{1} \mathrm{H}$ NMR $\left(\mathrm{CDCl}_{3}, 400 \mathrm{MHz}\right) \delta$ : 7.88 (d, $J=8.8 \mathrm{~Hz}, 2 \mathrm{H}), 7.70$ (d, $J=8.4 \mathrm{~Hz}, 2 \mathrm{H}), 7.65$ (s, $1 \mathrm{H}), 7.33 \sim 7.38(\mathrm{~m}, 5 \mathrm{H}), 4.56(\mathrm{~d}, J=5.6 \mathrm{~Hz}, 2 \mathrm{H}), 4.15(\mathrm{~s}$, $3 \mathrm{H}) ;{ }^{13} \mathrm{C} \mathrm{NMR}\left(100 \mathrm{MHz}, \mathrm{CDCl}_{3}\right) \delta: 161.3,158.3,145.2$, $138.1,136.9,135.8,134.5,132.3,129.4,129.2,128.8$, 127.7, 125.6, 107.2, 41.2, 35.6. Anal. calcd for $\mathrm{C}_{21} \mathrm{H}_{15} \mathrm{Cl}_{3}$ $\mathrm{N}_{4} \mathrm{O}_{2}$ : C 54.63, H 3.27, N 12.13; found C 54.52, H 3.12, N 12.30 .

4-氯-1-甲基-3-(2,4-二氟苯基)- $N$-[(5-苯基噁唑-2-基) 甲基]- $1 H$-吡唑-5-甲酰胺(11n): 白色固体，产率 51\%. m.p. $175 \sim 177{ }^{\circ} \mathrm{C} ;{ }^{1} \mathrm{H}$ NMR $\left(\mathrm{CDCl}_{3}, 400 \mathrm{MHz}\right) \delta: 7.63 \sim$ $7.65(\mathrm{~m}, 2 \mathrm{H}), 7.57(\mathrm{~s}, 1 \mathrm{H}), 7.41 \sim 7.52(\mathrm{~m}, 3 \mathrm{H}), 7.31 \sim$ $7.35(\mathrm{~m}, 2 \mathrm{H}), 6.92 \sim 7.00(\mathrm{~m}, 2 \mathrm{H}), 4.87(\mathrm{~d}, J=5.2 \mathrm{~Hz}$, 2H), $4.26(\mathrm{~s}, 3 \mathrm{H}) ;{ }^{13} \mathrm{C} \mathrm{NMR}\left(100 \mathrm{MHz}, \mathrm{CDCl}_{3}\right) \delta: 163.5$ $(\mathrm{d}, J=238.1 \mathrm{~Hz}), 161.6,159.3,158.3,152.3,142.3,132.4$, $131.4,129.0,128.8,127.5,124.3,121.8,114.9,111.6(\mathrm{~d}$, $J=21.2 \mathrm{~Hz}), 109.6,104.5,41.4$, 37.1. Anal. calcd for $\mathrm{C}_{21} \mathrm{H}_{15} \mathrm{ClF}_{2} \mathrm{~N}_{4} \mathrm{O}_{2}$ : C 58.82, H 3.53, N 13.07; found C 58.68, $\mathrm{H} 3.70, \mathrm{~N} 13.22$.

4-氯-1-甲基-3-(2,4-二氯苯基)- $N$ - \{[5-(4-氟苯基)噁 唑-2-基]甲基 $\}-1 H$-吡唑-5-甲酰胺(110): 白色固体，产率 52\%. m.p. $154 \sim 156{ }^{\circ} \mathrm{C} ;{ }^{1} \mathrm{H}$ NMR $\left(\mathrm{CDCl}_{3}, 400 \mathrm{MHz}\right) \delta$ : $7.97(\mathrm{~d}, J=2.00 \mathrm{~Hz}, 1 \mathrm{H}), 7.70 \sim 7.73(\mathrm{~m}, 1 \mathrm{H}), 7.50 \sim 7.63$ (m, 4H), $7.25(\mathrm{~s}, 1 \mathrm{H}), 7.11 \sim 7.15(\mathrm{~m}, 2 \mathrm{H}), 4.86(\mathrm{~d}, J=5.2$ $\mathrm{Hz}, 2 \mathrm{H}), 4.24(\mathrm{~s}, 3 \mathrm{H}) ;{ }^{13} \mathrm{C} \mathrm{NMR}\left(100 \mathrm{MHz}, \mathrm{CDCl}_{3}\right) \delta$ : $162.8(\mathrm{~d}, J=247.8 \mathrm{~Hz}), 159.3,158.2,151.5,144.0,135.8$, $132.7,132.2,130.8,130.5,129.8,129.2,127.1,126.6$, $126.2(\mathrm{~d}, J=8.2 \mathrm{~Hz}), 123.8,121.5,116.2(\mathrm{~d}, J=22.0 \mathrm{~Hz})$, 107.6, 41.4, 37.1. Anal. calcd for $\mathrm{C}_{21} \mathrm{H}_{14} \mathrm{Cl}_{3} \mathrm{FN}_{4} \mathrm{O}_{2}$ : C 52.58, H 2.94, N 11.68; found C 52.46, H 2.80, N 11.84 .

辅助材料(Supporting Information) 目标化合物 11a 110 的核磁共振氢谱与碳谱谱图. 这些材料可以免费从 本刊网站(http://sioc-journal.cn/)上下载.

\section{References}

[1] Phatangare, K. R.; Borse, B. N.; Padalkar, V. S.; Patil, V. S.; Gupta, V. D.; Umape, P. G.; Sekar, N. J. Chem. Sci. 2013, 125, 141.

[2] Liu, S. H.; Ling, Y.; Yang, X. L. Chin. J. Struct. Chem. 2013, 32, 931.

[3] Divya, K. R. G.; Sowmya, D. V.; Durgamma, S.; Tharanath, V.; Gopal, D. V. S.; Kumar, M. V. J.; Rao, C. A.; Padmaja, A.; Padmavathi, V. Med. Chem. Res. 2017, 26, 2568.

[4] Zhang, D. W.; Guo, J. M.; Zhang, M.; Liu, X.; Ba, M. Y.; Tao, X. Y.; Yu, L. Y.; Guo, Y.; Da, J. G. J. Nat. Prod. 2017, 80, 3242.

[5] Sangi, D. P.; Meira, Y. G.; Moreira, N. M.; Lopes, T. A.; Leite, M. 
P.; Pereira-Flores, M. E.; Alvarenga, E. S. Pest. Manage. Sci. 2019, 75, 262.

[6] Singh, I.; Rani, R.; Luxami, V.; Paul, K. Eur. J. Med. Chem. 2019, 166, 267.

[7] Chiacchio, M. A.; Lanza, G.; Chiacchio, U.; Giofre, S. V.; Romeo, R.; Iannazzo, D.; Legnani, L. Curr. Med. Chem. 2019, 26, 7337.

[8] Guo, J. C.; Hao, Y. A.; Ji, X. F.; Wang, Z. W.; Liu, Y. X.; Ma, D. J.; Li, Y. Q.; Pang, H. L.; Ni, J. P.; Wang, Q. M. J. Agric. Food Chem. 2019, 67, 10018.

[9] Wang, M. M.; Zhang, Q. Q.; Yue, K.; Li, Q. S.; Xu, F. B. Chin. J. Org. Chem. 2017, 37, 1774 (in Chinese) (王梦梦, 张青青, 岳凯, 李庆山, 徐凤波, 有机化学, 2017, 37, 1774.)

[10] Shi, Y. J.; Du, X. C.; Wang, X. L.; Chen, Q. W.; Li, L.; Dai, H.; Xu, C. Q.; Zhang, J. Y.; Ling, Y. Chin. J. Org. Chem. 2018, 38, 1772 (in Chinese).

(石玉军, 杜显超, 王祥龙, 陈庆文, 李玲, 戴红, 徐蔡芹, 张敬 远，凌勇，有机化学, 2018, 38, 1772.)

[11] Zhou, Qian.; Zheng, D. D.; Shi, Y. J.; Yao, W.; Qian, H. W.; Ding, Y.; Wei, Z. H.; Shen, A. B.; Feng, X.; Shi, J.; Dai, H. Chin. J. Org. Chem. 2018, 38, 3318 (in Chinese).

(周钱, 郑丹丹, 石玉军, 姚炜, 钱宏炜, 丁颖, 魏中昊, 沈爱宝, 冯霞, 石健, 戴红, 有机化学, 2018, 38, 3318.)

[12] Liu, Y. Y.; Li, Y.; Chen, N. Q.; Lü, K. Z.; Zhou, C.; Xiong, X. H.; Li, F. S. Molecules 2014, 19, 8140.

[13] Xiao, J. J.; Liao, M.; Chu, M. J.; Ren, Z. L.; Zhang, X.; Lü, X. H.; Cao, H. Q. Molecules 2015, $20,807$.

[14] Chen, K.; Liu, Q.; Ni, J. P.; Zhu, H. J.; Li, Y. F.; Wang, Q. Pest. Manage. Sci. 2015, 71, 1503.

[15] Huo, J. Q.; Ma, L. Y.; Zhang, Z.; Fan, Z. J.; Zhang, J. L.; Beryozkina, T. V.; Bakulev, V. A. Chin. Chem. Lett. 2016, 27, 1547.

[16] Liu, Q.; Zhu, R.; Gao, S.; Ma, S. H.; Tang, H. J.; Yang, J. J.; Diao, Y. M.; Wang, H. L.; Zhu, H. J. Pest. Manage. Sci. 2017, 73, 917.

[17] Iqbal, J.; Ejaz, S. A.; Saeed, A.; Al-Rashida, M. Eur. J. Pharmacol. 2018, 832,11 .
[18] Verma, G.; Chashoo, G.; Ali, A.; Khan, M. F.; Akhtar, W.; Ali, I.; Akhtar, M.; Alam, M. M.; Saquiquzzaman, M. Bioorg. Chem. 2018, $77,106$.

[19] Deng, X. L.; Zhou, X. M.; Wang, Z. Y.; Rui, C. H.; Yang, X. L. Chin. J. Struct. Chem. 2018, 37, 551.

[20] Jiang, B. B.; Jin, X. Y.; Dong, Y. W.; Guo, B. B.; Cui, L.; Deng, X. L.; Zhang, L.; Yang, Q.; Li, Y. X.; Yang, X. L.; Smagghe, G. J. Agric. Food Chem. 2020, 68, 6347.

[21] Yang, J. Y.; Tian, Y. R. Modern. Agrochem. 2002, (2), 11 (in Chinese). (杨建瑜，田永仁，现代农药, 2002, (2), 11.)

[22] Fan, W. Z.; Gu, B. Q.; Zhu, W. Q.; Zhang, Y. B. Modern. Agrochem. 2005, (4), 9 (in Chinese). (范文政，顾保权，朱伟清，张一宾，现代农药, 2005, (4), 9.)

[23] Chai, B. S.; Lin, D.; Liu, Y. X.; Liu, C. L. Chin. J. Pestic. 2007, 46, 148 (in Chinese). (柴宝山，林丹，刘远雄，刘长令，农药, 2007, 46, 148.)

[24] Liu, J. B.; Li, Y. X.; Zhang, X. L.; Hua, X. W.; Wu, C. C.; Wei, W.; Wan, Y. Y.; Cheng, D. D.; Xiong, L. X.; Yang, N.; Song, H. B.; Li, Z. M. J. Agric. Food Chem. 2016, 64, 3697.

[25] Wang, B. L.; Zhu, H. W.; Li, Z. M.; Wang, L. Z.; Zhang, X.; Xiong, L. X.; Song, H. B. Pest. Manage. Sci. 2018, 74, 726.

[26] Dai, H.; Yao, W.; Fang, Y.; Sun, S. Y.; Shi, Y. J.; Chen, J.; Jiang, G. Q.; Shi, J. Molecules 2017, 22, 2000.

[27] Wei, F.; Zhao, B. X.; Huang, B.; Zhang, L.; Sun, C. H.; Dong, W. L.; Shin, D. S.; Miao, J. Y. Bioorg. Med. Chem. Lett. 2006, 16, 6342.

[28] Wang, X. R.; Lin, X. H.; Xu, X. Q.; Li, W.; Hao, L. J.; Liu, C. C.; Zhao, D. M.; Cheng, M. S. Molecules 2017, 22, 1925.

[29] Zhu, Y.; Zheng, D. D.; Ni, Y. D.; Li, J. F.; Zhou, H. Y.; Hu, L. P.; Li, L.; Ju, J. F.; Chen, J. Z.; Li, H.; Shi, Y. J.; Dai, H. Chin. J. Org. Chem. 2020, 40, 774 (in Chinese).

(朱玥，郑丹丹，倪亚丹，李金峰，周环宇，胡兰萍，李玲，鞠剑 峰，陈家正，李宏，石玉军，戴红，有机化学, 2020, 40, 774.) 